“( 2015 IEEE. Personal use of this material is permitted. Permission from IEEE must be obtained for all other uses, in any current or future media, including reprinting/republishing this material for advertising or promotional purposes, creating new collective works, for resale or redistribution to servers or lists, or reuse of any copyrighted component of this work in other works." 


\title{
A Dynamic Almost Blank Subframe Scheme for Video Streaming Traffic Model in Heterogeneous Networks
}

\author{
A. Daeinabi, K. Sandrasegaran, and Sh. Barua \\ Centre for Real-time Information Networks, Faculty of Engineering and Information Technology, \\ University of Technology, Sydney,
}

Australia

\begin{abstract}
In heterogeneous network (HetNet), the coverage area of picocell is small due to transmission power difference between macro eNodeB (eNB) and pico eNB. As a result, the traffic load is distributed unequally which yields to macrocell overloading. In order to overcome this issue, cell range expansion (CRE) technique has been proposed. However, the CRE approach can affect the downlink signal quality of the offloaded users and then these users experience high downlink interference from macro eNB on their control and data channels. Therefore, such inter-cell interference coordination (ICIC) techniques are needed to realize the promised capacity and coverage. Enhanced ICIC (eICIC) is a time domain technique to mitigate interference in HetNets using almost blank subframes (ABSs). However, the static ABS value cannot support the dynamic changing of network conditions. In this paper, a dynamic ABS scheme is proposed based on Genetic Algorithm to find the best ABS value and $A B S$ locations in a frame to mitigate interference problem between macrocell and picocells for video streaming traffic model. Exhaustive simulation results show that the proposed scheme can improve the system performance in terms of throughput, outage probability and delay.
\end{abstract}

Keywords_ Heterogeneous network, intercell interference, picocell, cell range expansion, almost blank subframes, genetic algorithm.

\section{INTRODUCTION}

In recent years, data traffic demand is exponentially growing in in Long Term Evolution-Advanced (LTE-A) networks; therefore, more spectral efficiency is needed to support this demand. A flexible and scalable technique is the utilization of heterogeneous network (HetNet) consisting of macrocells and low power nodes such as femto, pico, and relay nodes [1]. The goal of using low power nodes is to offload the traffic from the macrocells, enhance coverage and throughput, and increase the spectral efficiency by spatial reuse of spectrum.

Picocell is one of the important low power nodes which can be deployed efficiently in local regions with high volume of traffic. This is because the picocell can improve the overall system capacity and coverage of outdoor or indoor regions with inadequate macro penetration [2]. However, using picocells can lead to new challenges for network management. One challenge is cell selection technique. Because of transmission power difference between macro eNodeB (eNB) and pico eNB, cell selection based on the strongest downlink Reference Signal Received Power (RSRP) is not the best strategy. That is because a User Equipment (UE) is connected to a higher power node instead of the lower power nodes at the shortest pathloss distance as shown in Fig.1 (a). This technique causes severe uplink interference between macro UEs and pico UEs [3]. One solution is Cell Range Expansion (CRE) in which an offset value is added to RSRP received from pico eNB such that the UE preferentially selects a pico eNB as the serving cell even when it is not the strongest cell as shown in Fig.1 (b). Although the CRE significantly mitigates interference in the uplink, the downlink signal quality of UEs located in the range expanded area (RE UE) decreases. Note that range expanded area is an area around a picocell where UEs are connected to pico eNBs because of receiving RSRP plus an offset value as shown in Fig.1 (b). Such RE UEs may suffer from downlink Signal to Interference plus Noise Ratio (SINR) below $0 \mathrm{~dB}$ because they are connected to cells that do not have the best downlink RSRP. Therefore, in the range expanded area, both data and control channel experience high interference which causes picocells become underutilized. Consequently, one of the important aspects of HetNets is interference management.

Enhanced Intercell Interference Coordination (eICIC) is a time domain technique to mitigate the interference problem using subframe utilization. This utilization is performed across different cells through Almost Blank Subframe (ABS) [4] which are subframes without any activity or only transmitting the reference signals from macro eNB. Pico eNB schedules RE UEs within subframes where overlap with the ABSs of the macrocell. Moreover, pico eNB can also transmit to its UEs during non-ABS periods. In other words, $\mathrm{ABS}$ configuration illustrates how picocells are informed about the interference pattern from the macro eNB and then schedule RE UE on subframes to avoid interference. Several eICIC schemes have been recently proposed to mitigate interference problem in time domain. Reference [5] has provided a good survey on 


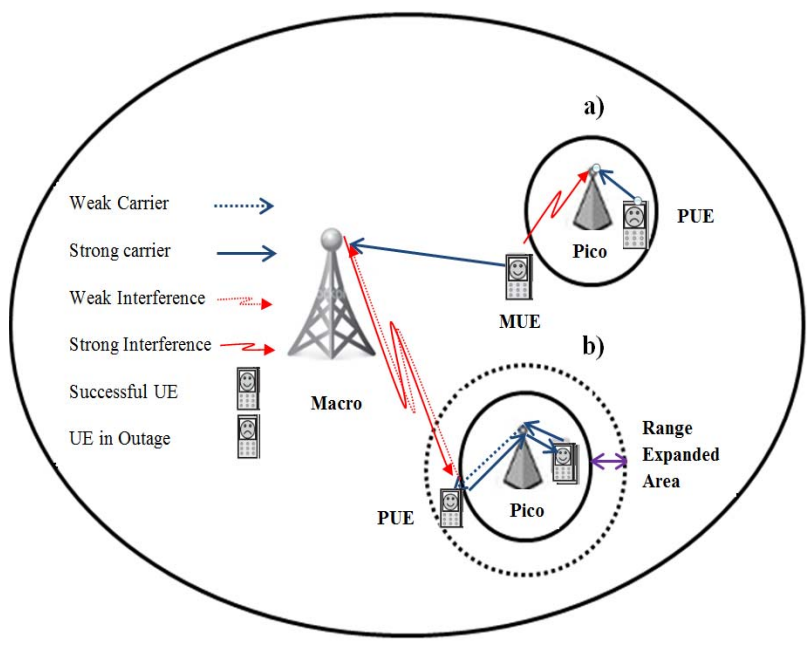

Fig.1. (a) Macro UE (MUE) interferes on the uplink of a nearby picocell; (b) Using cell range expansion to mitigate pico uplink interference

ICIC in LTE and LTE-Advanced. A dynamic ABS scheme proposed in [6] which used a logarithmic utility function based on total throughput of cell which is the sum of macrocell, edge and centre throughput of picocell. A subsequent dynamic ABS scheme was proposed in [7] where computed the number of ABSs dynamically based on the macro load, pico load and pico CRE load and using a minimization approach. However, if the number of ABSs is not set precisely, the throughputs of macro UEs and pico UEs are significantly degraded because the number of $\mathrm{ABS}$ and non-ABS are not corresponding to number of RE UEs and macro UEs. It is important that ABS value must be appropriately selected based on the required UE throughputs in both macrocell and picocells not only the total cell throughput. The reason is that the total cell throughput cannot guarantee that the required throughputs for all UEs are satisfied or not. Moreover, these schemes have not determined the location of ABSs in each frame.

This paper proposes a dynamic ABS scheme in time domain based on Genetic Algorithm (GA) when macrocell and picocells share the bandwidth. The proposed scheme aims to find the best ABS value and its configuration while maximizes system throughput and minimizes the delay and downlink interference between macrocell-picocells. GA is used to satisfy the requirements of video streaming traffic model because GA can handle multiple solutions during each iteration and avoid local sub-optima in search space.

The rest of the paper is organized as follows. Section II describes the network model. The multi-objective problem is explained in Section III. Section IV proposes the new eICIC scheme based on GA. Section V contains the simulation results and their discussion. The conclusion is given in the final section.

\section{NETWORK MODEL}

The network consists of macrocells and picocells which can be classified in terms of transmission powers, antenna heights, and the type of access mode provided for UEs. Macro eNBs are conventional operator-installed eNBs and usually cover a wide area of a few kilometres [8]. Picocells are used with lower transmission power than macrocells with smaller coverage area [9]. Picocells and macrocells usually work in open access mode where any UE in the overlapped coverage area can automatically be connected to either eNBs.

\section{A. User Association}

In this work, each UE selects its serving cell using (1) where an offset value is added to the RSRP received from pico eNB.

Servingcell_ID $=\arg \max _{\{m\}}\left(R S R P_{m, k}+\right.$ offset_value $)$

Different offset value in range $(020] \mathrm{dB}$ [10] can be added to RSRP received by $\mathrm{UE}_{k}$ from $\mathrm{eNB}_{m}$. When the offset value is set to $0, \mathrm{CRE}$ acts as the maximum RSRP technique. Note that the offset value is only added to RSRP received from the pico eNB. The offset value is broadcast by the cell to help UEs make the accurate association decision.

\section{MULTI-OBJECTIVE PROBLEM}

In this paper, some requirements of the video streaming traffic are considered as the objective functions including throughput, interference, delay and packet loss error (PLR). By using this metrics, the system performance can be monitored until the system reaches to an optimal performance. These objective functions aim to maximize the throughput while minimizing the interference, delay and PLR. Minimizing the PLR can guarantee the reliability of transmission while minimizing the delay guarantees packet delay requirements for on-going video streaming. Moreover, level of interference is kept low between macrocell and picocells by minimizing the interference while the maximization of throughput guarantees the minimum required data rates of macro UEs and pico UEs are satisfied.

\section{A. Maximizing Throughput}

The objective function of throughput can be given by (2)

$$
T=\frac{1}{N_{u}} \frac{1}{T_{s}} \sum_{k=1}^{N_{u}} \sum_{t=1}^{T_{s}} R_{i}(t)
$$

where $R_{i}(t)$ is the total size of the received packets (in bits) of $\mathrm{UE}_{k}$ at time $t, T_{s}$ is the total simulation time and $N_{u}$ is the total number of UEs in a macrocell and its picocells. Since a tradeoff between macro and pico UE throughputs is needed to optimize the performances of macrocell and picocell, we define two sub-functions:

$$
\begin{aligned}
& f_{1}=\frac{1}{R_{m}^{*}}\left(\frac{1}{N_{m}} \frac{1}{T_{s}} \sum_{k=1}^{N_{m}} \sum_{t=1}^{T_{s}} R_{i}(t)\right) \\
& f_{2}=\frac{1}{R_{p}^{*}}\left(\frac{1}{N_{p}} \frac{1}{T_{s}} \sum_{k=1}^{N_{p}} \sum_{t=1}^{T_{s}} R_{i}(t)\right)
\end{aligned}
$$

where $f_{1}$ and $f_{2}$ are the objective function of throughputs for macrocell and picocell, $N_{m}$ and $N_{p}$ represent the total number of macro UEs and pico UEs, respectively. $R_{m}^{*}$ and $R_{p}^{*}$ indicate the minimum required data rate of macro UEs and pico UEs, respectively. 


\section{B. Minimizing Interference}

The interference between macrocell and picocells can limit the throughputs particularly for RE UEs. At the minimizing approach, the objective function of the interference level on pico UEs is obtained as follows:

$$
f_{3}=\frac{1}{N_{p}} \sum_{k=1}^{N_{p}} \delta_{k} \frac{P_{k} H_{k}}{P_{l} H_{l}}
$$

where $P_{l}$ and $P_{k}$ are transmission power of serving $\mathrm{eNB}_{l}$ and interfering $\mathrm{eNB}_{k}$ on Physical Resource Block $n\left(\mathrm{PRB}_{n}\right)$, respectively. $H_{l}$ and $H_{k}$ present channel gain of serving $\mathrm{eNB}_{l}$ and interfering $\mathrm{eNB}_{k}$ on $\mathrm{PRB}_{n}$, respectively. $\delta_{k}$ is set to 1 or 0 to indicate whether the interfering cell $k$ allocates $\mathrm{PRB}_{n}$ to its UEs or not. Note that PRB is the smallest resource that can be allocated to a UE in LTE network.

\section{Minimizing PLR}

In the wireless network, PLR is defined as the percentage of the discarded packets of a service. Minimizing the PLR is one of the most important functions in real time services which can guarantee the reliability of packet transmission. Note that, the PLR has to be kept below a threshold in order to satisfy the QoS requirement.

$$
f_{4}=1-\frac{1}{P^{*}}\left(\frac{1}{N_{u}} \sum_{k=1}^{N_{u}} \frac{p_{d}}{p_{s}}\right)
$$

where $p_{d}$ is the total size of discarded packets (in bits) of $\mathrm{UE}_{k}$ and $p_{s}$ is the total size of all packets (in bits) arrived into the eNB's buffer of $\mathrm{UE}_{k} . P^{*}$ is the maximum packet loss tolerance for video streaming traffic. $F_{4}$ indicates whether average PLRs of all UEs is lower than the maximum packet loss tolerance.

\section{Minimizing Delay}

Delay is a subsequent factor affecting system performance of video streaming traffic. If the packet is not received by $\mathrm{UE}_{k}$ within a delay threshold it will be lost. Delay can be defined as the average delay of Head-of-Line (HOL) packets of all UEs. The HOL packet of a UE is the packet that has the longest resided time in the buffer of eNB.

$$
f_{5}=1-\frac{1}{D^{*}}\left(\arg \max _{u \in N_{u}}\left(\max \left(d_{p, k}\right)\right)\right)
$$

where $d_{p, k}$ is the delay of the $p^{\text {th }}$ packet of $\mathrm{UE}_{k}$ and $D^{*}$ is the maximum packet delay tolerance for video streaming traffic. $F_{5}$ indicates whether maximum packet delay of the system is lower than the maximum packet delay tolerance.

\section{E. Optimizing Multi-Objectives}

In multi-objectives optimization problem, the problem is how to find an optimal solution such that all desired objectives can be taken into account. One of the most popular and simplest approaches to solve multi-objective optimization problem is Weighted Sum Method [11], where multiple objective functions are combined into a single composite function using (8):

$$
f(x)=\sum_{i=1}^{5} w_{i} f_{i}(x)
$$

where $w_{i} \geq 0$ represents the relative importance of the objectives and it is assumed that $\sum_{i=1}^{j} w_{i}=1, f_{i}(x)$ is desired objective functions and $\left[x_{1}, x_{2}, \ldots, x_{j}\right]$ are the vector of decision variables. It is clear that the results are changed when the weighting coefficients change, hence the weighting coefficients can be allocated to objective functions corresponding to their roles in improvement of the system performance. Based on this approach, systems can instantaneously change their operating aims by adjusting the weighting coefficients.

\section{THE PROPOSED ABS SCHEME USING GENETIC ALGORITHM}

GA is one type of the population-based search algorithms in which the optimized solution is obtained using recombination and selection strategies. GA can be used to optimize the objective functions without much mathematical requirements. In GA, the specified variables of a problem can be symbolized as genes in a chromosome and the chromosomes are evaluated based on fitness values including some objective functions. For this purpose, a set of chromosomes are randomly selected as the initial population to encode a set of possible solutions. For the initialized population, some genes may show better performance in one chromosome while at the same time another chromosome may have some genes that are better than others. In order to combine best genes from different chromosomes, the crossover and mutation operators are used to produce new chromosomes named offspring. Finally, GA converges to the best solution after creating several generations. The GA is terminated when the termination condition is satisfied. The proposed ABS scheme aims to find the number of ABSs and their best locations in each frame.

\section{A. Population Initialization}

The initial population is obtained by randomly initializing variables. In order to reduce the computer memory overhead and computation cost, the population is scaled in a small size. In our proposed algorithm, the population is shown in Fig.(2) where each frame distribution is coded as one chromosome $(c h)$ and indicates which subframes $\left(S_{j}\right)$ are identified as ABS. In Fig.2, 1 indicates the subframes that are labelled as ABS and 0 indicates the subframes used as non-ABSs. For example, $C h_{n}$ represents that $\left\{S_{1}, S_{2}, S_{3}, S_{5}, S_{7}, S_{8}, S_{9}\right\}$ are non-ABSs while $\left\{S_{4}, S_{6}, S_{10}\right\}$ are used as ABSs.

\begin{tabular}{|c|c|c|c|c|c|c|c|c|c|c|}
\cline { 2 - 12 } \multicolumn{1}{c|}{} & $\mathrm{S}_{1}$ & $\mathrm{~S}_{2}$ & $\mathrm{~S}_{3}$ & $\mathrm{~S}_{4}$ & $\mathrm{~S}_{5}$ & $\mathrm{~S}_{6}$ & $\mathrm{~S}_{7}$ & $\mathrm{~S}_{8}$ & $\mathrm{~S}_{9}$ & $\mathrm{~S}_{10}$ \\
\hline $\mathrm{Ch}_{1}$ & 1 & 0 & 0 & 1 & 1 & 1 & 0 & 1 & 0 & 0 \\
\hline $\mathrm{Ch}_{2}$ & 0 & 1 & 1 & 1 & 0 & 0 & 1 & 1 & 1 & 1 \\
\hline
\end{tabular}

\begin{tabular}{|l|l|l|l|l|l|l|l|l|l|l|}
\hline $\mathrm{Ch}_{\mathrm{n}}$ & 0 & 0 & 0 & 1 & 0 & 1 & 0 & 0 & 0 & 1 \\
\hline
\end{tabular}

Fig.2. Initialized population 


\section{B. Fitness Evaluation}

Each chromosomes represented in the population has a corresponding fitness value. The fitness value is directly related to the objective function and used to determine which chromosomes will be selected for the next generation. The chromosome with the greater fitness value has more chances to be selected. In this paper, the weighted sum of five objective functions, $f(x)$, is considered as the fitness value.

\section{Crossover Operator}

For each generation, the crossover has an important role to find the optimal solution. The crossover helps genes of parents are combined and then passed to their offsprings. Moreover, it may produce new offsprings to expose the new search area. In this paper, single-point is used as the crossover operation where parents are divided into two parts in a single-point and then offsprings are generated by combining of first part of one parent and second part of another parent as shown in Fig. 3 (a) where $\left\{S_{1}, S_{2}, S_{3}, S_{4}\right\}$ of parent 1 are combined with $\left\{S_{5}, S_{6}, S_{7}\right.$, $\left.S_{8}, S_{9}, S_{10}\right\}$ of parent 2 and vice versa.

\section{Mutation Operator}

In order to find the optimal solution and avoid falling into the suboptimal solution, the mutation operation is required in which some modification is performed to provide possible variation in the offspring. Mutation operation changes one or more genes in a chromosome of parent to prevent the population from stagnating at any local optimum. Using a normal distribution, one or more gens are selected and then its value is changed as shown in Fig. 3 (b) where the value of $S_{4}$ and $S_{9}$ were changed using mutation operator.

\section{E. Selection}

The selection step determines which of the chromosomes will be kept for the next generation. It guaranties that the better chromosomes have more chance to be selected. In this paper, the roulette wheel method is used as the selection operator. The first step of selection is to compute the cumulative fitness of the total population using the sum of the fitness of all chromosomes. Then, probability of each chromosome is computed using (9) given as follows:

$$
P\left\{x_{i}\right\}=\frac{f\left(x_{i}\right)}{\sum_{j=1}^{m_{c h}} f\left(x_{j}\right)}
$$

where $m_{c h}$ is number of chromosomes and $f(x)$ is the fitness value of each chromosome. Thereafter, an array is constructed using cumulative probabilities of the chromosomes. Moreover, a vector is generated containing $n$ normalised random numbers. Finally, the surviving chromosomes will be selected by comparing the elements of these two vectors based on the $P\left\{x_{i}\right\}$.

\section{F. Termination}

A termination criterion needs to be set for GA to stop its iterating. The termination condition can be selected as the total number of iterations, maximum computing time, or a threshold of desired fitness values. In the proposed scheme, the termination condition is set to total number of iterations.

\section{SIMULATION RESULTS AND DISCUSSION}

This section evaluates the proposed scheme using MATLAB system level simulation. The simulation parameters are given in Table I based on parameters specified by 3GPP [9]. The traffic is modelled as video streaming service of a 256 kbps source video rate in this paper. Video traffic is modelled as a sequence of frames containing a fixed number of packets which arrive at a regular interval. The size of a packet and the inter-arrival time between two consecutive packets in each frame are assumed to be based on Truncated Pareto distribution [12].

\section{A. Trends of $A B S$ Value}

The percentage of ABSs in each frame and ABS configuration obtained by the proposed ABS scheme are presented for different CRE offset values in Table II. Observation demonstrates that the best ABS values are $10 \%$, $50 \%$ and $70 \%$ for CRE offset values of $9 \mathrm{~dB}, 12 \mathrm{~dB}$ and 15 $\mathrm{dB}$, respectively. This is because the requirement of system is satisfied for ABS values based on the defined fitness values. Moreover, the locations of ABSs are shown for different CRE offset values in Table II. In addition, Fig. 4 shows the convergence of the fitness values of different offset values.

\section{B. Throughput Performance}

In general, when the offset value is low (i.e., $9 \mathrm{~dB}$ ), the macro UEs throughput is low due to large number of UEs located in macrocell while using the large offset value leads to improve the macro UE throughput as shown in Fig.5. On the other hand, although using large offset value can improve the macro UE throughput, the throughput of RE UEs decreases due to low SINR experienced. In case of using ABS, when the number of the offloaded UEs is large the small ABS value cannot support all RE UEs and hence some of them are scheduled on non-ABS where experience high interference. In contrast, if a large ABS value is used for small offset value, number of non-ABS cannot satisfy the required macro UE throughput because of large number of macro UEs. Based on

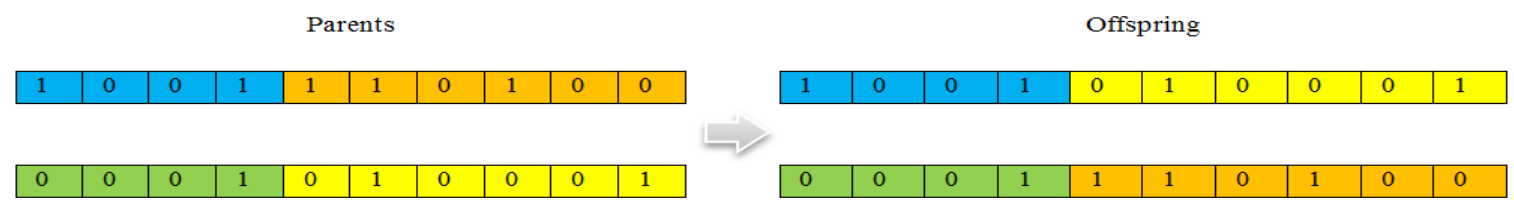

(a) Crossover

\begin{tabular}{|l|l|l|l|l|l|l|l|l|l|}
\hline 1 & 0 & 0 & 1 & 1 & 1 & 0 & 1 & 0 & 0 \\
\hline
\end{tabular}$-$\begin{tabular}{|l|l|l|l|l|l|l|l|l|l|l|}
\hline 1 & 0 & 0 & 0 & 1 & 1 & 0 & 1 & 1 & 0 \\
\hline
\end{tabular}

(b) Mutation

Fig.3. Examples of (a) crossover and (b) mutation operators 
Table I. Simulation parameters

\begin{tabular}{ll}
\hline \hline Parameter & Value \\
\hline Cellular Layout & $\begin{array}{l}\text { Hexagonal grid, } 7 \text { cells, wrap } \\
\text { around } \\
400 \mathrm{~m}\end{array}$ \\
Inter-site distance & $75 \mathrm{~m}$ \\
Minimum distance between macro & \\
and pico & $2 \mathrm{GHz}$ \\
Carrier frequency & $5 \mathrm{MHz}$ \\
Carrier bandwidth & $4 \mathrm{picos} / \mathrm{macro}$ \\
Pico distribution & $43 \mathrm{dBm}$ \\
Transmission power of macro eNB & $30 \mathrm{dBm}$ \\
Transmission power of pico eNB & $100 \mathrm{TTI}$ \\
Time window of ABS value & $150 \mathrm{~ms}$ \\
$\mathrm{D}^{*}$ & $10^{-3}$ \\
$\mathrm{P}^{*}$ & $250 \mathrm{Kbps}$ \\
$\mathrm{R}_{\mathrm{p}}{ }^{*}$ & $250 \mathrm{Kbps}$ \\
$\mathrm{R}_{\mathrm{m}}$ & 0.20 \\
$\mathrm{w}_{\mathrm{i}}(\mathrm{i}=1, \ldots, 5)$ & \\
\hline \hline
\end{tabular}

the simulation results shown in Figs.5 and 6, the proposed scheme can keep the balance between the macro UE and RE UE throughputs by deploying the optimum ABS value and configuration for video streaming traffic.

\section{Delay}

When the ABS configuration and offset value change, the number of PRBs allocated to each UE changes. For different SINR, an appropriate Modulation and Coding Scheme (MCS) is selected which results in a changing throughput. For example, for low SINR, a lower order modulation is used which provides a lower transmission bit rate. Therefore, if the number of the allocated PRBs and the selected MCS cannot support the amount of the received data, data are transmitted with delay. Since delay is considered as the one of the fitness metrics, the proposed scheme generates offspring by considering the delay. Fig.7 shows that the proposed scheme has an acceptable delay for difference CRE offset values while the other requirements of system are satisfied.

\section{Outage Probability}

Figure 8 illustrates the outage probability for schemes with different offset values. Note that outage probability is the ratio of UEs that their average SINR is smaller than the SINR threshold set to $-6.5 \mathrm{~dB}$ [13]. It is clear that the outage probability is an increasing function of offset value. That is because UEs far away from pico eNBs are offloaded to picocell by increasing of offset value while they suffer severe interference from macro eNB. Consequently, the probability that the RE UEs' SINR becomes smaller than the outage threshold increases. Fig. 8 shows that the outage probability is

Table II. ABS Configuration Obtained by GA based Scheme for Difference CRE Offset Values

\begin{tabular}{|c|c|c|c|c|c|c|c|c|c|c|c|}
\hline $\begin{array}{c}\text { Offset } \\
\text { Value }\end{array}$ & $\begin{array}{c}\text { ABS } \\
\text { Value }\end{array}$ & \multicolumn{10}{|c|}{ ABS configuration } \\
\hline $\mathbf{9} \mathbf{d B}$ & $10 \%$ & 0 & 0 & 0 & 1 & 0 & 0 & 0 & 0 & 0 & 0 \\
\hline $\mathbf{1 2} \mathbf{~ d B}$ & $50 \%$ & 1 & 0 & 1 & 0 & 1 & 0 & 0 & 0 & 1 & 1 \\
\hline $\mathbf{1 5} \mathbf{d B}$ & $70 \%$ & 0 & 0 & 1 & 1 & 0 & 1 & 1 & 1 & 1 & 1 \\
\hline
\end{tabular}

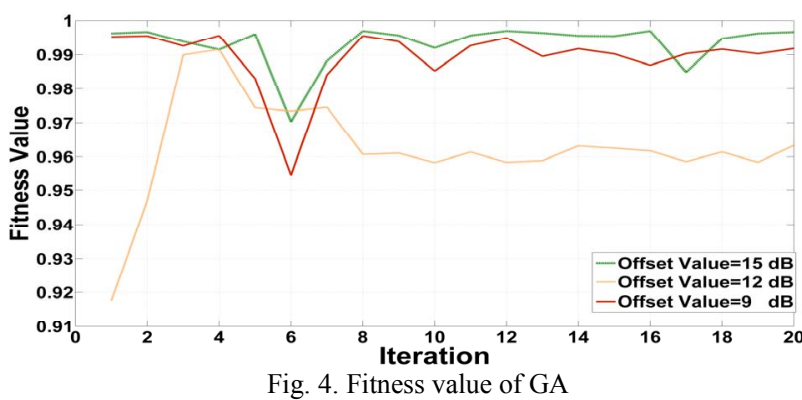

at a good level when video streaming traffic is used. This is because the proposed scheme considers the throughput of RE UEs when the ABS is configured.

\section{E. System Performance Comparison}

In order to evaluate the results of the proposed GA based scheme, the system performances of different eICIC schemes are compared in Table III for CRE offset value of $9 \mathrm{~dB}$ and video streaming traffic. The reason of comparison for $9 \mathrm{~dB}$ is that [6] selected the offset value of $9 \mathrm{~dB}$ as its optimum offset value. Therefore, all schemes are compared for $9 \mathrm{~dB}$ to compare results under the same situation.

Based on this table, the proposed GA base scheme and [6] converged to ABS value of $10 \%$ and $20 \%$ respectively, whereas [7] oscillated between 50\%-60\%. However, it is clear that the proposed scheme could keep the trade-off between macro UE and RE throughputs while [7] sacrificed the macro UE throughput to increase the RE UE throughput. Moreover, the proposed scheme outperforms [7] in terms of outage and delay. This is because the proposed GA based scheme considered the throughput of RE UEs, macro UE throughout, interference level and delay to find the best ABS configuration.

Although the GA based scheme and [6] obtained the similar results for macro UE and RE UE throughputs, the scheme proposed in [6] takes time much more than our proposed scheme to find the best ABS because it calculates the total throughput for all combination of (ABS value, offset value) and then selects the combination that obtains the maximum total throughput. Moreover, [6] only used the total sum of macro cell and pico cell throughputs to find the best ABS value. Therefore, it is possible the obtained total cell throughput for a particular combination of ABS and offset values becomes large because only the macro cell throughput or pico cell throughput is high while the RE UE throughput or macro UE throughput is sacrificed. Table III shows that the proposed scheme has lower delay than [6].

\section{CONCLUSION}

This paper proposed a ABS scheme based on GA approach to overcome interference problem in heterogeneous networks when macrocell and picocells share the bandwidth. The proposed scheme used the GA to intelligently select the optimum ABS value and the best location of ABSs in a frame. By considering the macro UE throughput, RE UE throughput, interference, delay and PLR, an excellent balance can achieve between the macro UE and RE UE throughputs. The system 


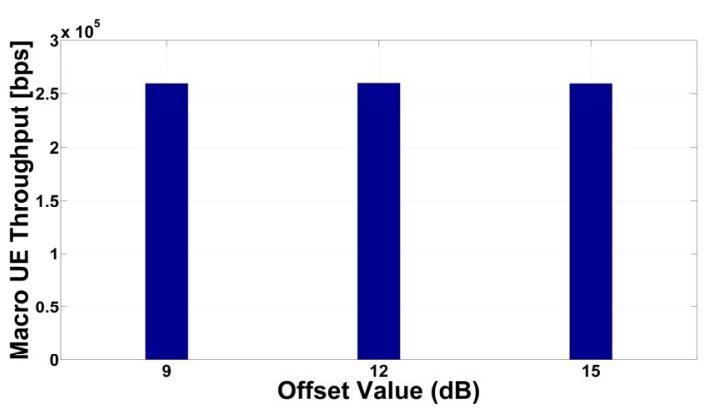

Fig. 5. Average macro UE throughput for difference CRE offset values

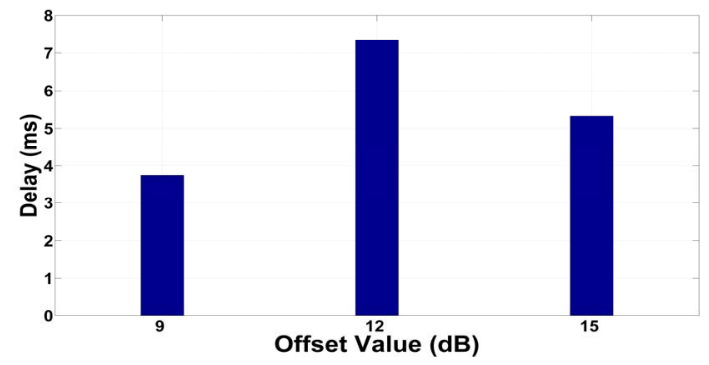

Fig .7. The delay obtained for difference CRE offset values

level simulation has been developed to support the analysis for video streaming traffic model. Simulation results showed the required throughput of macro UEs and RE UEs are guaranteed by the proposed scheme while the delay and outage probability are at an acceptable level.

\section{REFERENCES}

[1] A. Khandekar, N. Bhushan, J. Tingfang, and V. Vanghi, "LTE-Advanced: Heterogeneous Networks", European Wireless Conference, 2010.

[2] M. Shirakabe, A.Morimoto,and N. Miki, " Performance Evaluation of Inter-cell Interference Coordination and Cell Range Expansion in Heterogeneous Networks for Downlink LTE-Advanced," $8^{\text {th }}$ International Symposium on Wireless Communication Systems (ISWCS), pp. 844-848, November 2011.

[3] D. Lopez-Perez, I. Guvenc, G. de la Roche, M. Kountouris, T. Q. S. Quek, and Z. Jie, "Enhanced Intercell Interference Coordination Challenges in Heterogeneous Networks," IEEE Wireless Communications, vol.18, no.3, pp.22-30, June 2011.

[4] 3GPP TS 136.300, "Evolved Universal Terrestrial Radio Access (EUTRA) and Evolved Universal Terrestrial Radio Access Network (EUTRAN); Overall description; Stage 2 (Release 10),” 2011.

[5] Ch. Kosta, B. Hunt, A. U. Quddus and R. Tafazolli, "On Interference Avoidance Through Inter-Cell Interference Coordination (ICIC) based on OFDMA Mobile Systems," IEEE Communications Surveys \& Tutorials, vol. 15, no. 3, Third Quarter, pp. 973-995, 2013.

[6] M. Rawi, M. Simsek, R. Jantti, "Utility-based Resource Allocation in

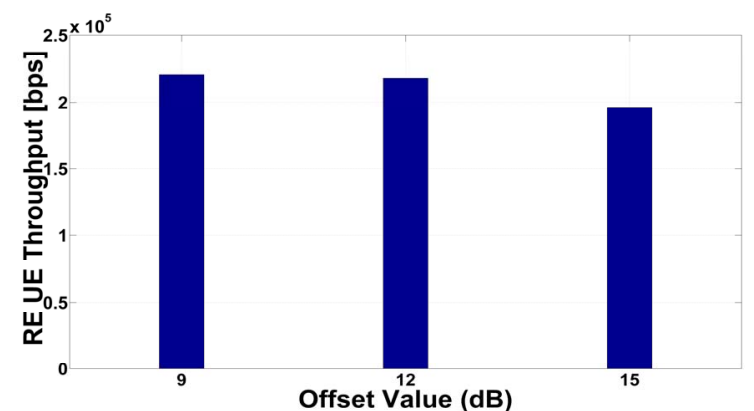

Fig. 6. Average RE UE throughput for difference CRE offset values

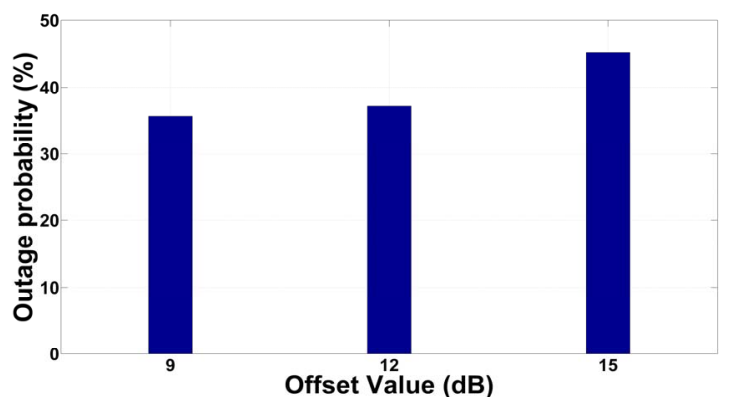

Fig. 8. The outage probability for difference CRE offset values

LTE-Advanced Heterogeneous Networks," $9^{\text {th }}$ International Wireless Communications and Mobile Computing Conference (IWCMC), Sardinia, July 2013,pp. $826-830$.

[7] S.N.S. Kshatriya, S. Kaimalettu, S.R. Yerrapareddy, K. Milleth, and N. Akhtar, "On Interference Management based on Subframe Blanking in Heterogeneous LTE Networks," Fifth International Conference on Communication Systems and Networks (COMSNETS), Bangalore, January 2013, pp.1-7.

[8] 3GPP, TS 36.814, "Evolved Universal Terrestrial Radio Access (EUTRA); Further Advancements for E-UTRA Physical Layer Aspects", March. 2010.

[9] 3GPP, TR 36.931, V10.0.0 “LTE; Evolved Universal Terrestrial Radio Access (E-UTRA); Radio Frequency Requirements for LTE Pico NodeB", May. 2011.

[10]K. Okino, T. N., C. Yamazaki, H. Sato, Y. Kusano, "Pico Cell Range Expansion with Interference Mitigation toward LTE-Advanced Heterogeneous Networks," IEEE International Conference on the Communications Workshops (ICC), pp. 1-5, June 2011.

[11] K. Deb, Multi-Objective Optimization using Evolutionary Algorithms Chichester [u,a]: Wiley, 2002.

[12] I.B. Aban, M.M. Meerschaert, and A.K. Panorska, "Parameter Estimation for the Truncated Pareto Distribution," Journal of the American Statistical Association, vol. 101, no. 473, March 2006, pp. 270-278

[13] 3GPP, R1-100350, "Downlink CCH Performance Aspects for CoChannel Deployed Macro and HeNBs," TSG RAN WG1 Meeting \#59bis, Nokia Siemens Networks, Nokia, January 2010.

Table III. System Performance Comparison for GA based Scheme

\begin{tabular}{|c|c|c|c|c|c|c|c|c|}
\hline \multirow[b]{2}{*}{ eICIC Scheme } & \multirow[b]{2}{*}{ Offset Value } & \multirow[b]{2}{*}{ ABS Value } & \multirow[b]{2}{*}{ Outage } & \multirow[b]{2}{*}{ Delay (ms) } & \multicolumn{4}{|c|}{ Throughput } \\
\hline & & & & & $\begin{array}{c}\text { Macro UE } \\
\text { (kbps) }\end{array}$ & $\begin{array}{c}\text { RE UE } \\
\text { (kbps) }\end{array}$ & $\begin{array}{c}\text { Macrocell } \\
\text { (Mbps) }\end{array}$ & $\begin{array}{l}\text { Picocell } \\
\text { (Mbps) }\end{array}$ \\
\hline GA based & $9 \mathrm{~dB}$ & 10 & $35.6 \%$ & 3.47 & 248 & 220 & 6.54 & 3.04 \\
\hline Reference [6] & $9 \mathrm{~dB}$ & 20 & $34.3 \%$ & 8 & 238 & 227 & 6.23 & 3.38 \\
\hline Reference [7] & $9 \mathrm{~dB}$ & $\begin{array}{l}\text { Oscillated } \\
\text { between } 50- \\
60\end{array}$ & $39.7 \%$ & 11.60 & 210 & 253 & 4.55 & 5.61 \\
\hline
\end{tabular}

